\title{
Profi-Mundhygiene schützt vor Pneumonie
}

\author{
Pneumonien sind nach wie vor eine der häufigsten Todesursachen bei älteren Menschen. Sie können \\ Ihre Patienten schützen, indem Sie ihnen eine professionelle zahnärztliche Mundpflege ermöglichen.
}

In einer Metaanalyse konzentrierten sich die Autoren auf die healthcareacquired pneumonia (HAP), die sowohl im Krankenhaus als auch im Pflegeheim viele Patienten betrifft. Multimorbidität und Polypharmazie sind als begünstigende Faktoren bekannt. Nun ging es um den Effekt einer assistierten Mundpflege auf das Mortalitätsrisiko.

Mittels einer systematischen Literaturrecherche wurden 2.678 Artikel zum Thema gefunden. Am Ende erfüllten fünf randomisierte, kontrollierte Studien die Einschlusskriterien. Sie wurden für die Metaanalyse herangezogen.

Es kam heraus, dass die Mortalität durch nosokomiale Lungenentzündung bei Patienten, deren Mundpflege von speziell zahnärztlich geschultem Perso- nal vorgenommen worden war, deutlich gesenkt werden konnte. Das relative $\mathrm{Ri}$ siko (RR) lag bei 0,43. In der Gruppe der Patienten, die vom regulären Personal oral gepflegt worden waren, konnte dieser Effekt hingegen nicht nachgewiesen werden $(\mathrm{RR}=1,20)$. Beide Pflegeverfahren wurden der sonst üblichen Pflege gegenübergestellt.

- Sjögren Pet al. Oral care and mortality in older adults with pneumonia in hospitals or nursing homes: systematic review and meta-analysis. J Am Geriatr Soc. 2016;64:2109-15

\section{KOMMENTAR}

Zu den Präventionsmaßnahmen gegen Pneumonien bei Älteren zählen neben Impfungen, ausgewogener Ernährung und körperlicher Aktivität auch die gute persönliche Hygiene und eine sorgfältige Mund- und Zahnpflege. So hat der
Zahnstatus, mit oder ohne prothetischem Ersatz, maßgeblich Einfluss auf eine mögliche Infektion. Hinzu kommen noch Schluckstörung und Dysphagie bei multimorbiden geriatrischen Patienten [Komiya Ket al. Aging Dis. 2014;6:27-37] und eine mögliche Besiedelung durch höher pathogene Keime bei Menschen mit deutlichen Funktionseinschränkungen [Wårdh IM, Wikström MB. Spec Care Dentist. 2014;34:64-9].

Zwar wird es sicherlich schwierig, zahnärztliches Fachpersonal für die Zahnund Mundpflege in Krankenhäusern und Pflegeheimen zu gewinnen, aber man sollte es angesichts der Tatsache, dass orale Hygiene ein bedeutender Schutzfaktor ist, auf jeden Fall versuchen.

Prof. Dr. med. H. J. Heppner

\section{Binnen drei Tagen wächst ein Tumor aus dem Auge}

Ein 30-jähriger Mann kam wegen einer gestielten, polypenartigen Gewebsvermehrung, die über den Rand des rechten unteren Augenlids heraushing, in eine Augenambulanz. Zwei Wochen zuvor hatte sich aus einer Zyste am selben Ort spontan Flüssigkeit entleert. Vor drei Tagen dann war eine kleine Vorwölbung der Bindehaut am Bulbus entstanden, die schnell gewachsen war. Verlauf und Untersuchungsbefund sprachen für ein pyogenes Granulom, eine gutartige Gefäßläsion, die durch Entzündungszellen und eine lobuläre kapilläre Proliferation gekennzeichnet ist.

Ein konjunktivales pyogenes Granulom kann nach einer Verletzung der Bindehaut - etwa durch Operation oder Trauma - innerhalb kurzer Zeit rasch an Größe zunehmen. Differenzialdiagnostisch kommen Nahtgranulome, Papillome und maligne Tumoren wie ein Plattenepithelkarzinom oder ein amelanotisches Melanom in Betracht. Pyogene Granulome sind oft vulnerabel und neigen zu Blutungen. Sie können entweder konservativ mit topischen Kortikoiden behandelt oder chirurgisch entfernt werden.

Im vorliegenden Fall injizierte man vor der chirurgischen Entfernung Triamcinolon in den Tumor, um das Risiko für ein Rezidiv zu verringern. Die histologische Untersuchung bestätigte die klinische Verdachtsdiagnose. Bei einer Kontrolluntersuchung drei

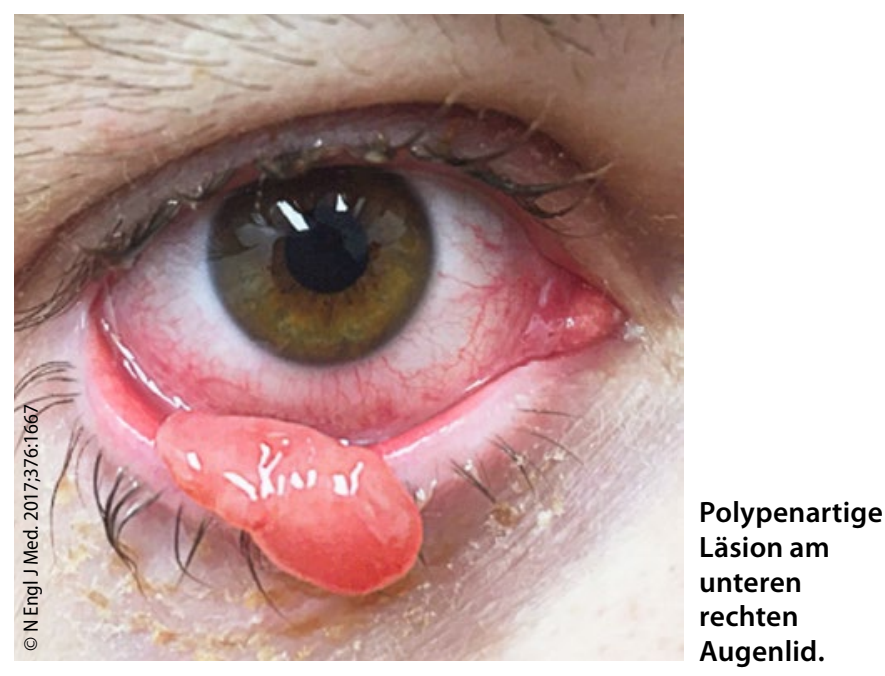

Monate später zeigte sich nur eine minimale Narbenbildung der konjunktivalen Oberfläche ohne Anhalt für ein Rezidiv.

Prof. Dr. med. H. S. FüeßI

- Tan IJ, Turner AW. Pyogenic granuloma of the conjunctiva. N Eng/ J Med. 2017;376:1667 